\title{
Production of SHLA-G molecules by in vitro matured cumulus-oocyte complex
}

\author{
ROBERTA RIZZO $^{1 *}$, MARIA BEATRICE DAL CANTO ${ }^{*}$, MARINA STIGNANI $^{1}$, RUBENS FADINI ${ }^{2}$, \\ DANIELA FUMAGALLI ${ }^{2}$, MARIO MIGNINI RENZINI ${ }^{2}$, MONICA BORGATTI $^{3}$, \\ ROBERTO GAMBARI ${ }^{3}$ and OLAVIO ROBERTO BARICORDI ${ }^{1}$ \\ ${ }^{1}$ Department of Experimental and Diagnostic Medicine, Laboratory of Immunogenetics, University of Ferrara, \\ Via Luigi Borsari, 46-44100 Ferrara; ${ }^{2}$ Biogenesi Reproductive Medicine Centre, Istituti Clinici Zucchi, \\ Via Zucchi, 24-20052 Monza; ${ }^{3}$ ER-GenTech, Department of Biochemistry and Molecular Biology, \\ University of Ferrara, Via Fossato di Mortara, 74-44100 Ferrara, Italy
}

Received March 3, 2009; Accepted May 25, 2009

DOI: 10.3892/ijmm_00000261

\begin{abstract}
Oocyte selection with the highest competence is a major goal in IVF. Several studies demonstrated that nonclassical HLA class I HLA-G molecule modulation creates a tolerogenic microenvironment at the feto-maternal interface and is implicated in embryo implantation. This study investigated if soluble HLA-G molecules producted by the cumulus-oocyte complex (COC) are markers of oocyte maturation. sHLA-G molecule levels were analyzed using Bio-Plex assay in 152 COC supernatants obtained from 42 women and maturated by an 'in vitro maturation procedure'. The presence of sHLA$\mathrm{G}$ molecules was confirmed by Western blotting technique. The results demonstrate detectable amounts of sHLA-G molecules ranging from 300 to $800 \mathrm{pg} / \mathrm{ml}$ in 14/73 (19\%) COCs that generated mature oocytes and complete absence of detectable sHLA-G antigens in the supernatants of COCs that corresponded to immature oocytes. The detection of sHLA-G molecules in the $\mathrm{COC}$ culture supernatants corresponding to
\end{abstract}

Correspondence to: Professor Roberto Gambari, ER-GenTech, Department of Biochemistry and Molecular Biology, University of Ferrara, Via Fossato di Mortara, 74-44100 Ferrara, Italy

E-mail: gam@unife.it

Dr Roberto Baricordi, Department of Experimental and Diagnostic Medicine, Laboratory of Immunogenetics, University of Ferrara, Via Luigi Borsari, 46-44100 Ferrara, Italy

E-mail: bri@unife.it

${ }^{*}$ Contributed equally

Abbreviations: HLA, histocompatibility antigen; IVF, in vitro fertilization; COC, cumulus-oocyte complex; FF, follicular fluids; IVM, in vitro maturation; $\mathrm{PCO}$, polycystic ovarian syndrome;

Key words: HLA-G, oocyte, in vitro maturation matured oocytes is proposed to be a marker to identify gametes with higher functionality. This non-invasive marker could be used, in addition to morphological approaches, to reduce the number of fertilized oocytes and transferred embryos.

\section{Introduction}

Currently, several oocytes are fertilized during in vitro fertilization (IVF) procedures and two or more embryos are transferred to the uterus in order to increase the chance of a pregnancy. However, this approach results in a high number of multiple pregnancies, perinatal mortality and morbidity. The necessity to overcome these risks together with the presence of ethical problems increases the interest in selecting gametes with the highest competency. This point is of extreme interest in Italy, where the law 40/2004 states that no more than three embryos can be created at any one time and all embryos obtained must be transferred together even if the couple does not need all the embryos (1). In this context the identification of validated markers for oocyte selection represents a fundamental step in IVF.

Several studies demonstrated that HLA-G antigen modulation creates, by direct and indirect mechanisms, a tolerogenic microenvironment at the feto-maternal interface (2). HLA-G molecules inhibit, together with HLA-C and -E, the innate natural killer response against cytotrophoblast cells which lack classical HLA class I and II expression (3-5). HLA-G antigens affect the adaptative cellular response inducing the apoptosis of cytotoxic $\mathrm{CD} 8^{+} \mathrm{T}$ lymphocytes $(6,7)$, impairing $\mathrm{CD} 4^{+} \mathrm{T}$ cell functions and preventing dendritic cell maturation $(8,9)$. HLA-G molecules also induce immunosuppressive regulatory T cell development $(10,11)$.

Detectable levels of soluble HLA-G (sHLA-G) molecules in a percentage of follicular fluids (FFs) from patients admitted to IVF procedures were recently reported (12) and granulosa cells were identified as producers of SHLA-G molecules. The granulosa cells which surround the mammalian oocyte are known as the cumulus oophorus. These cells create a structural pathway for cell-to-cell communication (13) where cumulus cells provide several trophic or metabolic factors for the 
Table I. Details of in vitro maturation procedure.

\begin{tabular}{lc}
\hline & $\begin{array}{c}\text { Women } \\
\mathrm{n}=42\end{array}$ \\
\hline Age (years) (mean \pm SD) & $35 \pm 3$ \\
Number of recovered oocytes & $7 \pm 1$ \\
per woman (mean \pm SD) & \\
Mature oocytes per woman $(\%)$ & $20-50$ \\
\hline
\end{tabular}

preovulatory oocyte (14). Several results indicate that the measurement of gene transcription levels in cumulus cells reliably complement the morphological oocyte evaluation providing a useful tool for selecting oocytes with greater chances to be fertilized $(15,16)$.

Considering the presence of sHLA-G in FFs during the oocyte maturation, this study verified if it is a marker of this process. The culture supernatants of 'in vitro matured' oocytes were analyzed for sHLA-G presence. The target of 'in vitro maturation' (IVM) technique is to retrieve immature oocytes from the ovary and to induce their maturation in vitro. We employed in vitro co-cultures of oocytes and cumulus cells to restore support from the surrounding cumulus cells to the oocyte $(17,18)$. This system allowed us to analyze the sHLA-G production by the cumulus-oocyte complex (COC) without the influence of the in vivo maternal microenvironment.

\section{Materials and methods}

Patients. The oocytes employed for this study were obtained from regularly cycling patients attending the Biogenesi Reproductive Medicine Centre of Monza, Italy, for an Assisted Reproduction Technique with In Vitro Maturation Protocol (IVM). Couples included in the trial had an indication of the IVF procedure because of infertility due to the male factor, tubal factor, stage I/II endometriosis, polycystic ovarian syndrome (PCO) or unexplained causes. All the women included had regular cycles of 26-35 days. A written informed consent was obtained from all participating couples. We considered just one cycle per couple. After the maturation process we used from one to three oocytes according to the Italian Law 40 on IVF (1). Following these criteria, 42 women were recruited for the study. Characteristics of the female patients are reported in Table I. Oocyte recovery was performed by means of transvaginal ultrasonound-guided follicle aspiration, using a single lumen aspiration needle (Gynetics cod. 4551-E2 Ø17-gauce $35 \mathrm{~cm}$ ) connected to a vacuum pump (Craft Pump pressure $80-90 \mathrm{mmHg}$ ). The aspirated FFs, containing COCs, were washed with prewarmed Flushing Medium with heparin (Medi-Cult product no. 10760125, Denmark). The oocytes were detected under a stereomicroscope, examined and classified on the basis of their morphology. Oocytes with signs of mechanical damage or atresia were discarded. Immature COCs were individually cultured in a 4-well culture dish with $0.5 \mathrm{ml}$ of IVM Medium (vial 2 of IVM system medium; Medicult no. 82214010, Denmark) supplemented with rec-FSH $0.075 \mathrm{IU} / \mathrm{ml}$ (Serono, Italy), hCG $0.1 \mathrm{IU} / \mathrm{ml}$ (Serono, Italy) and
Table II. Mature COC and oocyte grade scoring.

\begin{tabular}{llc}
\hline A, COC & & \\
\hline Mature COC grade & Cumulus & Adhesion to oocyte \\
\hline Grade A & $\begin{array}{l}\text { expanded } \\
\text { multilayer } \\
\text { full/spare } \\
\text { Grade B }\end{array}$ & full \\
& $1-3$ layers & spare \\
\hline
\end{tabular}

\section{B, Oocyte}

\begin{tabular}{lcc}
\hline Mature oocyte grade & Cytoplasmatic & Polar body \\
\hline Grade 1 & homogenous & round \\
Grade 2 & granular & round \\
Grade 3 & granular & fragmented/irregular
\end{tabular}

$10 \%$ serum protein substitute (SPS no. 3010, Sage Media, USA) for $30 \mathrm{~h}$. Following the maturation period, the supernatants were collected from each culture system containing a single COC and stored at $-20^{\circ} \mathrm{C}$ until tested for the presence of sHLA-G. The oocytes were denuded from cumulus and evaluated for the presence of the first polar body to confirm Metaphase II stage. The Metaphase II oocytes were considered mature, while the Metaphase I, degenerated and germinal vescicle oocytes were defined as immature (19). The mature oocytes were classified according to surrounding cumulus cells as follows. Grade A, expanded cumulus with multilayer and slack cumulus cells with a full adhesion to the oocyte with cumulus; Grade B, full or spare compact cumulus with one to three layers of cumulus cells with a spare adhesion to the oocyte (Table IIa); and graded on the first polar body and cytoplasm characteristics. Grade 1, homogeneous cytoplasm and round polar body; Grade 2, oocyte with variations in colour or granularity of the cytoplasm and/or presence of inclusions, vacuoles or retractable bodies, but a round polar body; Grade 3, oocyte with variations in colour or granularity of the cytoplasm and/or presence of inclusions, vacuoles or retractable bodies with a fragmented polar body (Table IIb).

sHLA-G ELISA. sHLA-G1, obtained from the proteolytic cleavage of the membrane bound HLA-G1 and -G5, generated by mRNA alternative splicing, was assayed as reported in Essen Workshop on sHLA-G quantification (20) using as capture antibody the Moab MEM-G9 (Exbio, Prague, Czech Republic), which recognizes HLA-G molecule, in $\beta_{2}$-microglobulin-associated form, at the concentration of $20 \mu \mathrm{g} / \mathrm{ml}$. The anti- $\beta_{2}$ microglobulin Moab-HRP conjugated, (Dako, Glostrup, Denmark) was used as detecting antibody diluted 1:1,000 in PBS1x. HeLa cell wild-type culture supernatants were used as negative control, transfected HeLa-G5 cell (kindly provided by Professor E. Weiss, Institut fur Anthropologie und Genetik, LMU, Munich, Germany) as a positive control. Culture supernatants were collected at cell confluence and concentrated by lyophilization procedure. Following depletion 
Table III. Calculated standard concentrations from ELISA and BioPlex assays.

\begin{tabular}{lcr}
\hline A, ELISA assay & & \\
\hline $\begin{array}{l}\text { Standard Conc } \\
\mathrm{pg} / \mathrm{ml}\end{array}$ & $\begin{array}{c}\text { Calculated Conc } \\
\mathrm{pg} / \mathrm{ml}\end{array}$ & $\begin{array}{c}\text { Error }^{\mathrm{b}} \\
\%\end{array}$ \\
\hline 800 & 1035 & 29.4 \\
500 & 535 & 7.0 \\
400 & 485 & 21.3 \\
300 & 435 & 45.0 \\
& Mean error $(\%)$ & 25.7 \\
\hline
\end{tabular}

\section{B, BioPlex assay}

\begin{tabular}{lcc}
\hline $\begin{array}{l}\text { Standard Conc } \\
\mathrm{pg} / \mathrm{ml}\end{array}$ & $\begin{array}{c}\text { Calculated Conc } \\
\mathrm{pg} / \mathrm{ml}\end{array}$ & $\begin{array}{c}\text { Error }^{\mathrm{b}} \\
\%\end{array}$ \\
\hline 800 & 798 & 0.3 \\
500 & 514 & 2.6 \\
400 & 379 & 5.0 \\
300 & 315 & 5.0 \\
200 & 195 & 3.0 \\
& & 3.2 \\
\hline
\end{tabular}

${ }^{a}$ For the calculated standard concentrations we used the regression curve equations: The $\mathrm{r}^{2}$ values were 0.9434 and 0.9958 for ELISA and

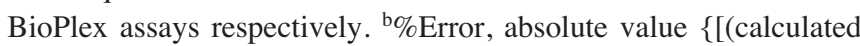
concentration-standard concentration)/standard concentration] $\mathrm{x} 100\}$.

of albumin by albumin depletion kit (Enchant Life Science kit, Pall Corporation, MI, USA), the purification of the sHLA-G proteins was carried out as previously reported (21). The sHLA-G molecules obtained were used as standard at different dilutions. COC culture medium was used for standard dilution. Detection limit was calculated with repeated measurements of a negative control obtained with culture medium. This way all the variables of the assay were considered. We calculated the mean of the optical density (OD) value obtained in the negative control wells, present in triplicate in each plate, and a standard deviation (SD), with the value of lower limit of detection being 3.29 SD added to the mean OD value (22). In this case, there is only a $5 \%$ chance of classifying a result in the wrong population and the lower limit of detection sample determinations are above this midway concentration with a probability of $95 \%$. The limit of sensitivity is $600 \mathrm{pg} / \mathrm{ml}$.

Bio-Plex system: covalent coupling of antibodies to microspheres. Covalent coupling of anti-sHLAG antibodies to the carboxylated polystyrene microspheres (Bio-Rad, Hercules, CA, USA) was performed using the Bio-Plex amine coupling kit (Bio-Rad). Briefly, the microspheres stock solution was dispersed by bath sonication until a homogeneous distribution of the microspheres was observed. For a 1x scale coupling reaction, $100 \mu \mathrm{l}$ of monodisperse beads $\left(1.25 \times 10^{6}\right.$ microspheres), were centrifugated at 14,000 x g for 4 min and washed with $100 \mu$ l of bead wash buffer. Bead pellets were resuspended in $80 \mu \mathrm{l}$ of bead activation buffer, vortexed for $30 \mathrm{sec}$, and then sonicated by bath sonication for $30 \mathrm{sec}$. Solutions of N-hydroxysulfosuccinimide (S-NHS) and 1-ethyl-3-(3-dimethylaminopropyl)-carbodiimide hydrochloride (EDC) (Pierce Biotechnology, Rockford, IL, USA), both at $50 \mathrm{mg} / \mathrm{ml}$, were prepared in bead activation buffer immediately prior to its use and $10 \mu \mathrm{l}$ of each solution was sequentially added to stabilize the reaction and activate the microspheres. This suspension was vortexed for $30 \mathrm{sec}$ and then agitated with a rotator at room temperature for $20 \mathrm{~min}$ in the dark. Then activated beads were washed twice with $150 \mu 1$ of PBS, pH 7.4, incubated with $5 \mu \mathrm{g}$ of Moab MEM-G9 (Exbio) in a final volume of $500 \mu \mathrm{l}$ of $\mathrm{PBS}, \mathrm{pH} 7.4$ and agitated with a rotator at $4^{\circ} \mathrm{C}$ overnight in the dark. After washing with $500 \mu 1$ of PBS, pH 7.4 beads were resuspended with $250 \mu 1$ of blocking buffer and agitated at room temperature for $30 \mathrm{~min}$ in the dark. Coupled beads were washed with $500 \mu 1$ of storage buffer, resuspended in $150 \mu \mathrm{l}$ of storage buffer and counted with a hemacytometer. Coupling efficiency of monoclonal antibodies was tested by staining 10,000 microspheres with a biotinylated antibody directed to the source of the capture antibody (goat anti-mouse immunoglobulin G, e-Bioscience, San Diego, USA) followed by streptavidin-PE for 30 and $10 \mathrm{~min}$ at room temperature in the dark respectively. Microspheres, resuspended in $150 \mu 1$ of storage buffer, were measured and analyzed with the Bio-Plex system (Bio-Rad).

s-HLAG Bio-Plex assay. COC culture supernatants were assayed for sHLA-G using a bead array system Bio-Plex (Bio-Rad) according to the manufacturer's instructions. Briefly, $50 \mu 1$ of sHLA-G standards (prepared in the same fresh culture medium and assayed in duplicate) or samples (COC culture supernatants in duplicate) incubated with $50 \mu 1$ of anti-sHLA-G conjugated beads (5000 beads/well) in 96-well filter plates for $60 \mathrm{~min}$ at room temperature with shaking. Plates were washed by vacuum filtration three times with $100 \mu 1$ of Bio-Plex wash buffer, $25 \mu 1$ of biotinylated antibody W6/32 $(10 \mu \mathrm{g} / \mathrm{ml})$ (Dako) was added, and plates were incubated for $30 \mathrm{~min}$ at room temperature with shaking. After three filter washes, $50 \mu 1$ of streptavidin-phycoerythrin was added, and the plates were incubated for $15 \mathrm{~min}$ at room temperature with shaking. Finally, plates were washed by vacuum filtration three times, beads were suspended in $125 \mu 1$ of Bio-Plex assay buffer, and samples were analyzed on the Instrument Bio-Plex system in combination with the Bio-Plex Manager software. The standard curves for sHLA-G were used from 24,000 to $93.75 \mathrm{pg} / \mathrm{ml}$ and the minimum detectable dose was $300 \mathrm{pg} / \mathrm{ml}$. The detection limit was calculated with repeated measurements of a negative control obtained with culture medium. We considered all variables of the assay. We calculated the mean of the fluorescence value obtained in negative control wells, present in triplicate in each plate, and $\mathrm{SD}$, with the value of lower limit of detection being 3.29 SD added to the mean FI (22). In this case, there is only a $5 \%$ chance of classifying a result in the wrong population and the lower limit of detection sample determinations are above this midway concentration with a probability of $95 \%$. The specificity 
of this assay was validated with an isotype control (Mouse IgG1 Isotype control, code 1B-457-C100 Biotin; Exbio) used in the place W6/32 Biotin Moab. The background observed was lower than the selected detection limit (data not shown). We obtained the limit of sensitivity at $300 \mathrm{pg} / \mathrm{ml}$.

Western blotting. The presence of HLA-G molecules in COC supernatants were analysed by denaturating SDS-PAGE in a group of ELISA sHLA-G positive and negative samples (12). Briefly, 100x concentrated and albumin depleted pooled COC supernatants were loaded on $10 \%$ SDS-polyacrylamide gel, electrophoresed at $80 \mathrm{~V}$ for $2 \mathrm{~h}$ and blotted onto PVDF membrane (Immobilon-P Millipore, Billerica, MA, USA) by electrotransfer at $100 \mathrm{~V}$ for $45 \mathrm{~min}$ in $25 \mathrm{mM}$ Tris Buffer, $190 \mathrm{mM}$ glycine, 2\% SDS and (v/v) methanol each. Blocking was carried out with $5 \%$ nonfat dry milk, Tris $100 \mathrm{mM} \mathrm{pH} \mathrm{7.5,}$ $\mathrm{NaCl} 150 \mathrm{mM}$ overnight at $4^{\circ} \mathrm{C}$. After two washes, the membrane was incubated with MEM-G1 Moab (Exbio) $(10 \mu \mathrm{g} /$ $\mathrm{ml}$ ), which recognizes HLA-G molecule in denaturated form, for $3 \mathrm{~h}$ at room temperature with gentle shaking. The sHLA-G molecules were detected using protein-G HRP (Bio-Rad) at dilution of 1:5,000 in $10 \mathrm{mM}$ Tris $\mathrm{pH} 8.0,150 \mathrm{mM} \mathrm{NaCl}, 0.1 \%$ Tween-20. Reactions were developed by chemiluminescence with SuperSignal enhanced chemiluminescence kit (Super Signal West Pico system, Pierce) and captured by Chemiluminescence Imaging Geliance 600 detection system (PerkinElmer, CT, USA). Soluble HLA-G molecules, purified as previously reported (sHLA-G ELISA section), were used as positive control, culture medium as negative control. The COC supernatants were loaded at the same total protein concentration that was evaluated by Quick Start Bradford protein assay (Bio-Rad). The molecular weights were determined with the BenchMark (Invitrogen, CA, USA) pre-stained protein ladder (range 10-200 kD).

Statistics. The Fisher's exact test was used to analyze the different frequencies in sHLA-G positivities between COCs that generated mature or immature oocytes. The Correlation $\mathrm{Z}$ and Regression tests were used to analyze the interpolation of ELISA and Bio-Plex standard curves. The statistical analysis was conducted using Stat View software package (SAS Institute Inc., Cary, NC, USA). P-value of $\leq 0.05$ was considered significant (two tailed).

\section{Results}

Comparison between the SHLA-G ELISA and Bio-Plex assays. We compared the Bio-Plex assay and ELISA technique in quantifying sHLA-G molecules. We used the same capture antibody and standard reagents in both techniques in order to maintain the same specificity. They were compared for their sensitivity by geometric dilutions of the sHLA-G standard reagent. In Fig. 1a and $\mathrm{b}$ the standard curves of the ELISA and Bio-Plex assays were obtained with $50 \mu \mathrm{l}$ of standard reagent at the concentrations of $800,500,400,300 \mathrm{pg} / \mathrm{ml}$. The sensitivity was calculated with repeated measurements of a negative control and a 3.29 SD was calculated (22) (see Materials and methods). We interpolated the data in order to obtain a standard curve. The low limit of detection, corresponding to the $3.29 \mathrm{SD}$ added to the mean FI or OD value of the negative control, reached $300 \mathrm{pg} / \mathrm{ml}$ in the Bio-Plex assay

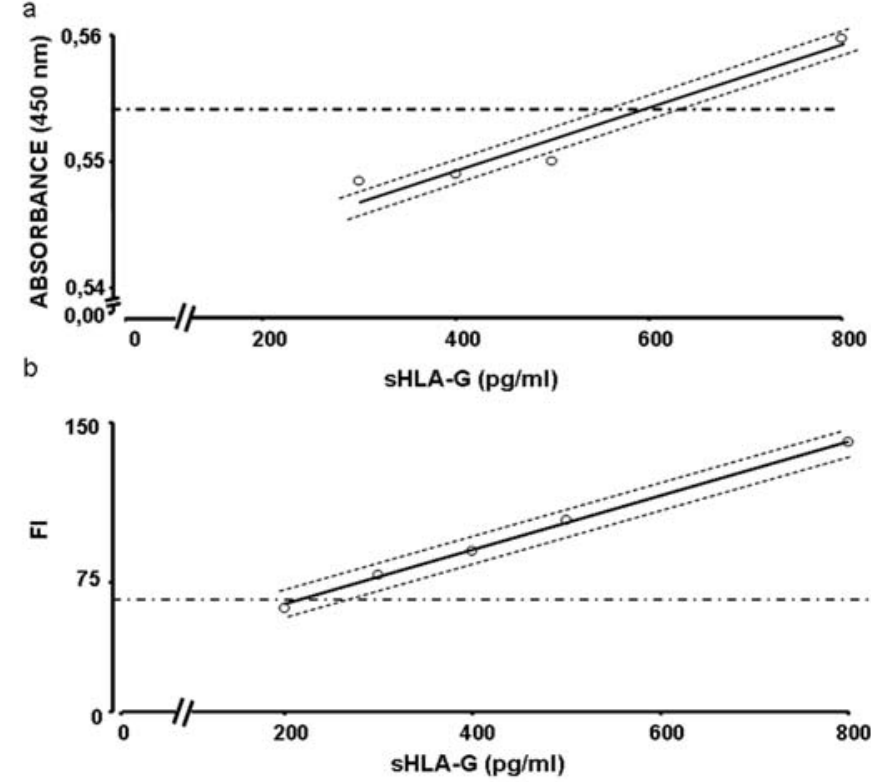

Figure 1. ELISA (a) and Bio-Plex (b) standard curves (black line). They were obtained with $50 \mu 1$ of standard reagent at the concentrations of 800 , $500,400,300 \mathrm{pg} / \mathrm{ml}$. Detection limit, dotted line, $3.29 \mathrm{SD}$ added to the mean optical density (OD) or fluorescence (FI) of repeated negative control (culture medium) measurements. The bold line indicates the linear regression and the dashed lines the $95 \%$ confidence interval. $\mathrm{r}^{2}$ Values, 0.9434 and 0.9958 for the ELISA and Bio-Plex assays respectively and CV values, 3.1 and 1.7 respectively.

and $600 \mathrm{pg} / \mathrm{ml}$ in the ELISA system. We observed a different accuracy in these two assays. As reported in Table III, the Bio-Plex calibration curve described the standard data with the lowest error (mean error, $3.2 \%$ ) in comparison to ELISA calibration curve (mean error, 25.7\%) (23). The coefficient of variation $(\mathrm{CV})$ for the Bio-Plex assay was 1.7 whereas the ELISA presented a CV of 3.1. The statistical analysis of these two calibration curves has a higher correlation in the Bio-Plex standard curve in comparison with ELISA (Correlation Z test, Bio-Plex $\mathrm{p}<0.0001, \mathrm{r}^{2}=0.9958$; ELISA $\mathrm{p}=0.035, \mathrm{r}^{2}=0.9434$; Regression test Bio-Plex $\mathrm{p}=0.0001$; ELISA $\mathrm{p}=0.0287$ ). The low $\mathrm{CV}$ error and detection limit associated with the Bio-Plex test indicated to select this assay to analyze the COC culture supernatants in order to reach lower sHLA$G$ levels with a higher degree of reliability in comparison with the ELISA system. The highest sensitivity of Bio-Plex assay is considered in comparison to our ELISA system and for low sHLA-G concentrations.

Detection of sHLA-G molecules in COC culture supernatants. We analyzed the COC culture supernatants by means of BioPlex technology. We analyzed 152 COC supernatants from IVM procedures performed in 42 women. Among the COCs, 73 matured (Metaphase II stage) and 79 remained immature (Prophase I or Metaphase I stage). Each woman had from one to three mature oocytes and from one to two immature oocytes. We revealed sHLA-G levels above $300 \mathrm{pg} / \mathrm{ml}$ in $14 / 73$ (19\%) COC supernatants that generated mature oocytes (Fig. 2), in a range from 309 to $800 \mathrm{pg} / \mathrm{ml}$, while no sHLA-G was detected in COC supernatants corresponding to immature oocytes ( $\mathrm{p}=8.4 \times 10^{-5}$; Fisher exact test). These results 




Figure 2. sHLA-G levels in $73 \mathrm{COC}$ culture supernatants that generated mature oocytes. Detection limit, dotted line, 3.29 SD added to the mean fluorescence (FI) of repeated negative control (culture medium) measurements.

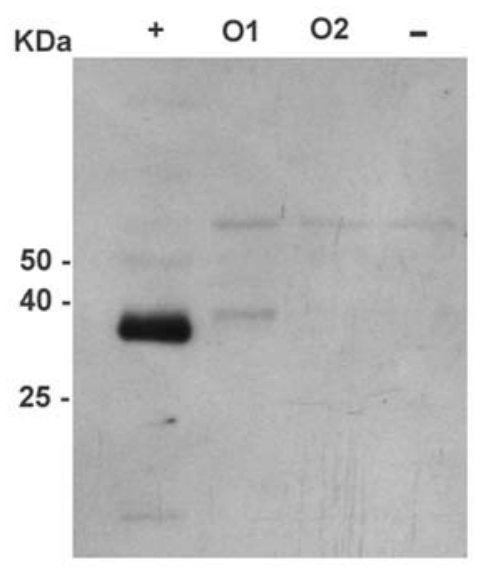

Figure 3. Western blot analysis of $\mathrm{COC}$ supernatants positive (O1) or negative (O2) for sHLA-G detection by Bio-Plex system. Anti HLA-G1/HLA-G5 MEM-G1 MoAb was used for detection. +, HLA-G purified molecules as positive control; -, culture medium as negative control.The COC supernatants were loaded at the same total protein concentration that was evaluated by Quick Start Bradford protein assay. The molecular weights (Mk) were determined with the BenchMark (Invitrogen, CA, USA) pre-stained protein ladder (range 10-200 kD).

document the presence of sHLA-G molecules only in COCs that produced mature oocytes.

Western blot analysis. We confirmed the data obtained in COC supernatants by Bio-Plex technique with Western blot analysis. In Fig. 3 we show a representative blot with a sHLA-G positive COC supernatant (second lane) and a sHLA-G negative COC supernatant (third lane), confirming the results obtained by Bio-Plex. We considered the upper bands present in all the samples as aspecific positivities and the $37 \mathrm{kD}$ positive band as HLA-G specific.

sHLA-G molecules and mature COC characteristics. The variability in SHLA-G levels could be associated with different oocyte characteristics. We evaluated the morphological feature of mature COCs (Table I). Taking into consideration the surrounding cumulus cell feature, we observed no differences in the percentage of sHLA-G positive supernatants in Grade A mature COCs (expanded cumulus) $(9 / 43 ; 21 \%$ ) in comparison to Grade B COCs (full or spare cumulus) $(5 / 30 ; 17 \%)(\mathrm{p}=\mathrm{NS}$, Fisher exact test) (Table IV; Fig. 4a). Considering the polar
Table IV. Subdivision of mature oocytes accordingly to COC and oocyte grading and sHLA-G production.

\begin{tabular}{lcc}
\hline & $\begin{array}{c}\text { Mature oocyte } \\
(\mathrm{n}=73)\end{array}$ & $\begin{array}{c}\text { sHLA-G+ } \\
(\mathrm{n}=14)\end{array}$ \\
\hline $\begin{array}{l}\text { COC grade } \\
\text { Grade A }\end{array}$ & 43 & 9 \\
Grade B & 30 & 5 \\
Oocyte grade & & \\
Grade 1 & 22 & 8 \\
Grade 2 & 15 & 1 \\
Grade 3 & 36 & 5 \\
\hline
\end{tabular}

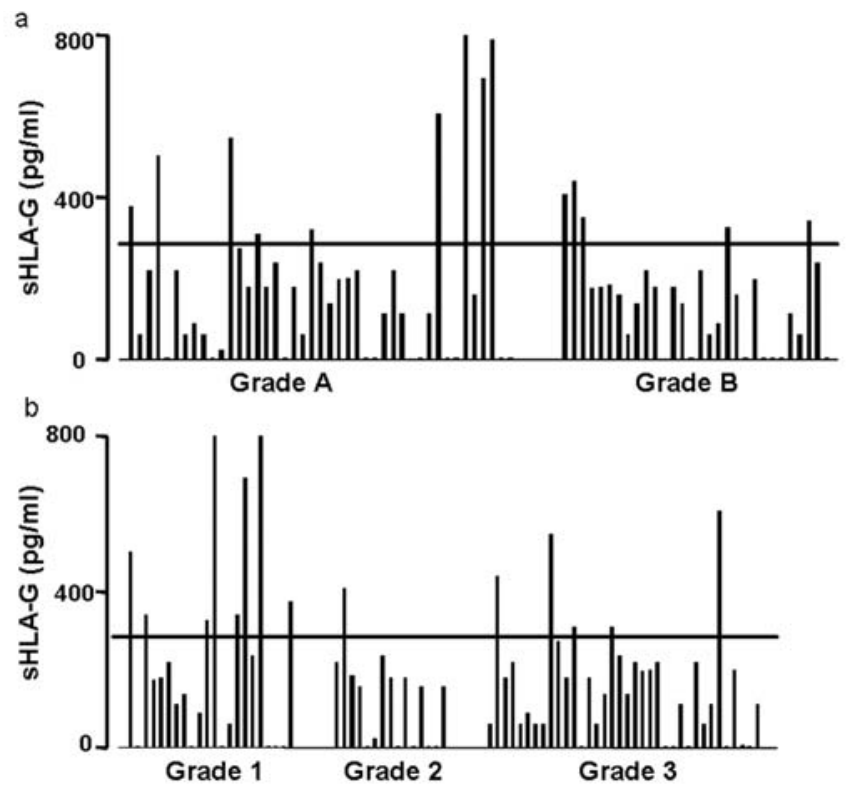

Figure 4. sHLA-G levels in mature COC supernatants subdivided accordingly to a) COC feature (Grade A and B); b) characterization of oocyte polar body and cytoplasm (Grade 1, 2 and 3). Detection limit, dotted line, 3.29 SD added to the mean fluorescence (FI) of repeated negative control (culture medium) measurements.

body and cytoplasmic morphological characteristics of mature oocytes (Metaphase II), evaluated after cumulus removal, we observed a difference in the percentage of sHLA-G positive COC supernatants which correspond to Grade 1 oocytes $(8 / 22 ; 36 \%)$ in comparison to Grade $2(1 / 15 ; 7 \%)$ $(\mathrm{p}=0.056$, Fisher exact test) and Grade $3(5 / 36 ; 14 \%)$ ( $\mathrm{p}=0.058$, Fisher exact test) (Table IV; Fig. 4b). The increased ability of COCs that generated Grade 1 oocytes to produce sHLA-G molecules is near the significant statistical p-value, probably because of the small number of samples. However, the clear tendency of mature COCs with high score oocytes to produce more sHLA-G molecules, proposes that these molecules are markers of oocyte grade. Therefore, increasing the number of analyzed COCs would confirm this observation.

sHLA-G molecules in mature COC culture supernatants subdivided between women. In order to identify a possible different sHLA-G production between the mature COCs of 

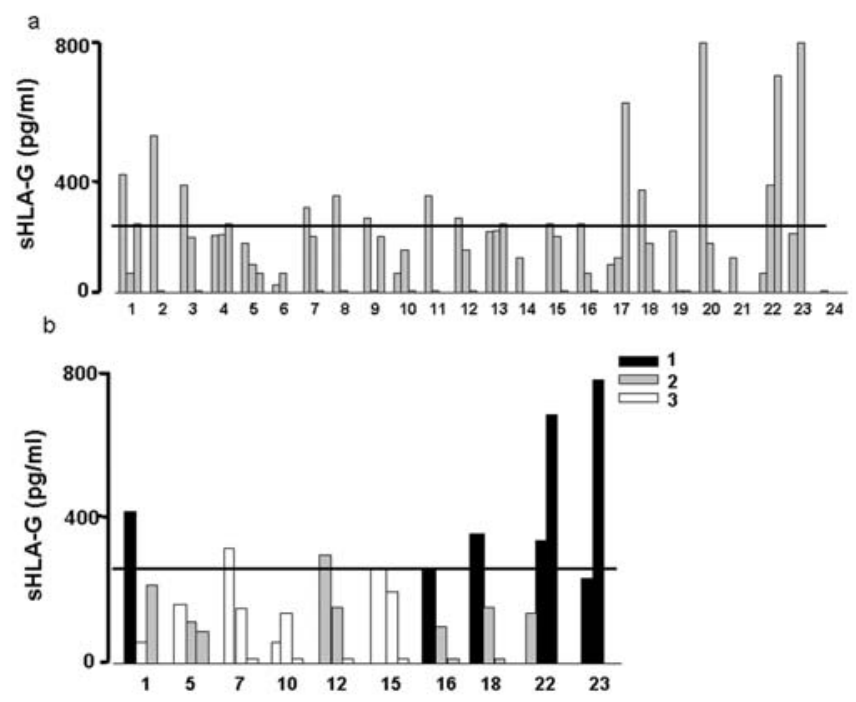

Figure 5. sHLA-G levels in mature COC supernatants subdivided into women (a) and in 10 representative women with oocyte Grade (grade 1, black; grade 2, grey and grade 3, white). Detection limit, dotted line, 3.29 SD added to the mean fluorescence (FI) of repeated negative control (culture medium) measurements.

the same woman, we subdivided them between women (Fig. 5a). The 14 supernatants with sHLA-G positivity corresponded to 13 different women. Taking into consideration the women with at least one sHLA-G positive COC culture, we observed different sHLA-G levels in their COC supernatant. For example, in Fig. 5a, woman number 1 presents two COC supernatants with no sHLA-G and one with $410 \mathrm{pg} / \mathrm{ml}$ of sHLA-G; woman number 10 has all three COC cultures negative for sHLA-G detection. The women presented different sHLA-G levels when we compared COC supernatants between women. These data are of great interest because COCs could be characterized by a different ability to produce HLA-G molecules. Taking into consideration the previous results on oocyte morphology and differences in sHLA-G modulation, we analyzed if the differences in sHLA-G levels between women correlate to different oocyte Grades. In Fig. 5b we show 10 representative women. There is an association between the differences observed in SHLA-G levels and the oocyte Grades. Woman number 1 has two COC supernatants without sHLA-G classified as Grade 3 (white), and Grade 2 (grey) and one with $410 \mathrm{pg} / \mathrm{ml}$ of sHLA-G Grade 1 (black); woman number 10 has all three COC cultures negative for sHLA-G and Grade 3 oocytes.

\section{Discussion}

The reduction of the number of fertilized oocytes and transferred embryos is the main target of assisted reproductive medicine. During recent years, several studies suggested a relationship between the production of sHLA-G molecules by early embryos and an increased implantation rate in IVF protocols $(24-35,12)$. Two studies $(36,37)$ failed to detect sHLA-G molecules in embryo culture supernatants, probably due to technical discrepancies, as suggested by the recent review by Warner et al (38). It is important to develop a very high quality level in sHLA-G detection methodology to overcome these problems in order to evaluate the exact amount of sHLA-G produced by an in vitro cultured embryo (39) and to establish the functional role of sHLA-G during early embryo development (40). However, data obtained by twelve researches suggest the production of sHLA-G as a marker of embryo implantation. It is still mandatory to evaluate embryo morphological parameters for an accurate embryo selection.

Oocytes obtained under ovarian stimulation present a variable competence and although molecular approaches have been proposed $(41,42)$ the selection is still performed on morphological characteristics. Recently, the presence of sHLA-G molecules in a percentage of FFs and a significant association with the production of these antigens by the corresponding fertilized oocyte was reported (12). Shaikly and coauthors (35) confirmed the presence of SHLA-G molecules in FFs but they failed to identify the correlation with early embryo sHLA-G production. Several differences in embryo culture conditions and in the technical procedures to detect sHLA-G could justify the different results obtained by these two studies. The presence of sHLA-G in FFs is not a confirmation that it is important in oocyte maturation but it seems to be a marker of embryo competency. However, further studies are required to confirm the relationship between FFs and embryo sHLA-G production. The goal of this study was to identify if sHLA-G is also a marker of oocyte maturation. The oocyte maturity was demonstrated to be important in producing good quality blastocysts for embryo transfer (19) and molecular markers. To define oocyte maturity would be of great interest together with morphological characterization. A sHLA-G assay based on Luminex technology reaching a detection limit of $300 \mathrm{pg} / \mathrm{ml}$ was proposed (34). We developed a similar Bio-Rad's Bio-Plex system reaching the same limit of sensitivity.

Our results show that COCs produce sHLA-G molecules during the oocyte maturation process. The main point is that no sHLA-G molecules were detected in the COC culture supernatants corresponding to immature oocytes. Some COCs produced mature oocytes but no sHLA-G was observed in their supernatants, underlining that SHLA-G is only one of the factors implicated in this process. Overall these results suggest a variable production of sHLA-G molecules in association with a different oocyte maturation. sHLA-G production was related to the morphological characteristics of mature COCs in order to analyze the possible functional role of these differences. All mature COCs were classified accordingly to their granulosa cell morphology, and to the oocyte cytoplasm and polar body characteristics before insemination. Taking into consideration the surrounding cumulus cell feature, we observed similar percentages of sHLA-G positive supernatants in Grade A and B mature COCs (Fig. 4a). On the contrary, the classification using polar body and cytoplasm characteristics demonstrated a tendency of Grade 1 mature oocytes to produce sHLA-G molecules higher than Grade 2 and 3 oocytes (Fig. 4b). This association proposes sHLA-G as a marker to identify high score mature oocytes. It is mandatory to increase the number of analyzed oocytes in order to confirm our observation based on a statistical significance near the p-value limit. The analysis of sHLA-G concentrations in mature COCs between women shows different sHLA-G levels both in mature COCs from the same woman and from different women. These data confirm 
that COCs are characterized by a different ability to produce sHLA-G molecules that is associated with the different oocyte grade.

Further analyses are required to define the mechanisms that influence sHLA-G production and the role of this antigen as directly implicated in oocyte maturation or as a marker of COC metabolism. A metabolic cooperation between oocyte and the surrounding granulosa cells is required for a complete maturation. The absence of sHLA-G molecules in immature COCs suggests a role for these molecules in oocyte maturation. It is known that cumulus cells express innate immune related genes (CD14, Toll-like receptors) (43) that play critical roles in surveillance and cell survival during the ovulation process and HLA-G could be one of these mechanisms. sHLA-G could be important in the maintenance of a balance between pro- and anti-inflammatory effectors and the absence of this molecule in COC supernatants could identify a difficulty in creating the correct maturation microenvironment.

In conclusion, these data demonstrate, for the first time, the ability of mature COCs to produce detectable amounts of sHLA-G molecules. This production could be a marker of good quality oocyte maturation and used to select the best oocyte to be fertilized in addition to the morphological approaches in order to reduce the number of produced and transferred embryos.

\section{Acknowledgements}

We thank Dr Amanda Neville for her writing assistance. R.G. is recipient of grants from Fondazione Cariparo (Cassa di Risparmio di Padova e Rovigo), CIB, UE eInfrastructure for the Thalassaemia Research Network (ITHANET) Project, Telethon (contract GGP07257), COFIN-2007, EU Project COCHISE and Associazione Veneta per la Lotta alla Talassemia (AVLT), Rovigo.

\section{References}

1. Fineschi V, Neri M and Turillazzi E: The new Italian law on assisted reproduction technology. J Med Ethics 31: 536-539, 2005.

2. Rouas-Freiss N, Naji A, Durrbach A and Carosella ED: Tolerogenic functions of human leukocyte antigen G: from pregnancy to organ and cell transplantation. Transplantation 84 S21-S25, 2007.

3. Moffett $A$ and Loke C: Immunology of placentation in eutherian mammals. Nat Rev Immunol 6: 584-594, 2006.

4. Khalil-Daher I, Riteau B, Menier C, Sedlik C, Paul P, Dausset J, Carosella ED and Rouas-Freiss N: Role of HLA-G versus HLA-E on NK function: HLA-G is able to inhibit NK cytolysis by itself. J Reprod Immunol 43: 175-182, 1999.

5. Rouas-Freiss N, Goncalves RM, Menier C, Dausset J and Carosella ED: Direct evidence to support the role of HLA-G in protecting the fetus from maternal uterine natural killer cytolysis. Proc Natl Acad Sci USA 94: 11520-11525, 1997.

6. LeGal FA, Riteau B, Sedlik C, Khalil-Daher I, Menier C, Dausset J, Guillet JG, Carosella ED and Rouas-Freiss N: HLA-G mediated inhibition of antigen-specific cytotoxic T lymphocytes. Int Immunol 11: 1351-1356, 1999.

7. Fournel S, Aguerre-Girr M, Huc X, Lenfant F, Alam A, Toubert A, Bensussan A and Le Bouteiller P: Cutting edge: soluble HLA-G1 triggers CD95/CD95 ligand-mediated apoptosis in activated CD8 ${ }^{+}$ cells by interacting with CD8. J Immunol 164: 6100-6104, 2000.

8. Bainbridge DR, Ellis SA and Sargent IL: HLA-G suppresses proliferation of CD4(+) T-lymphocytes. J Reprod Immunol 48: 17-26, 2000.

9. Lila N, Rouas-Freiss N, Dausset J, Carpentier A and Carosella ED: Soluble HLA-G protein secreted by allo-specific $\mathrm{CD} 4^{+} \mathrm{T}$ cells suppresses the allo-proliferative response: a CD4 $4^{+} \mathrm{T}$ cell regulatory mechanism. Proc Natl Acad Sci USA 98: 12150-12155, 2001.
10. Feger U, Tolosa E, Huang YH, Waschbisch A, Biedermann T, Melms A and Wiendl H: HLA-G expression defines a novel regulatory T-cell subset present in human peripheral blood and sites of inflammation. Blood 110: 568-577, 2007.

11. Selmani Z, Naji A, Zidi I, Favier B, Gaiffe E, Obert L, Borg C, Saas P, Tiberghien P, Rouas-Freiss N, Carosella ED and Deschaseaux F: Human leukocyte antigen-G5 secretion by human mesenchymal stem cells is required to suppress T lymphocyte and natural killer function and to induce $\mathrm{CD} 4{ }^{+} \mathrm{CD} 25$ highFOXP3 ${ }^{+}$ regulatory T cells. Stem Cells 26: 212-222, 2008.

12. Rizzo R, Fuzzi B, Stignani M, Criscuoli L, Melchiorri L, Dabizzi S, Campioni D, Lanza F, Marzola A, Branconi F, Noci I and Baricordi OR: Soluble HLA-G molecules in follicular fluid: a tool for oocyte selection in IVF? J Reprod Immunol 74: 133-142, 2007.

13. Gilchrist RB, Ritter LJ and Armstrong DT: Oocyte-somatic cell interactions during follicle development in mammals. Anim Reprod Sci 82-83: 431-446, 2004.

14. Elvin JA, Clark AT, Wang P, Wolfman NM and Matzuk MM: Paracrine actions of growth differentiation factor-9 in the mammalian ovary. Mol Endocrinol 13: 1035-1048, 1999.

15. Feuerstein P, Cadoret V, Dalbies-Tran R, Guerif F, Bidault R and Royere D: Gene expression in human cumulus cells: one approach to oocyte competence. Hum Reprod 22: 3069-3077, 2007.

16. Cillo F, Brevini TA, Antonini S, Paffoni A, Ragni G and Gandolfi F: Association between human oocyte developmental competence and expression levels of some cumulus genes. Reproduction 134: 645-650, 2007.

17. Combelles CM, Cekleniak NA, Racowsky C and Albertini DF: Assessment of nuclear and cytoplasmic maturation in in-vitro matured human oocytes. Hum Reprod 17: 1006-1016, 2002.

18. Eppig JJ: Intercommunication between mammalian oocytes and companion somatic cells. Bioessays 13: 569-574, 1991.

19. Huang FJ, Huang HW, Lan KC, Kung FT, Lin YC, Chang HW and Chang SY: The maturity of human cumulus-free oocytes is positively related to blastocyst development and viability. J Assist Reprod Genet 19: 555-560, 2002.

20. Rebmann V, Lemaoult J, Rouas-Freiss N, Carosella ED and Grosse-Wilde H: Report of the Wet Workshop for Quantification of Soluble HLA-G in Essen. Human Immunol 66: 853-863, 2005.

21. LeFriec G, Laupeze B, Fardel O, Sebti Y, Pangault C, Guilloux V, Beauplet A, Fauchet R and Amiot L: Soluble HLA-G inhibits human dendritic cell-triggered allogeneic T-cell proliferation without altering dendritic differentiation and maturation processes. Hum Immunol 64: 752-761, 2003.

22. Anderson DJ: Determination of the lower limit of detection. Clin Chem 35: 2152-2153, 1989.

23. Plikaytis BD, Turner SH, Gheesling LL and Carlonem GM: Comparisons of standard curve-fitting methods to quantitate Neisseria meningitides group A polysaccharide antibody levels by enzyme-linked immunosorbent assay. J Clin Microbiol 29: 1439-1446, 1991.

24. Fuzzi B, Rizzo R, Criscuoli L, Noci I, Melchiorri L, Scarselli B, Bencini E, Menicucci A and Baricordi OR: HLA-G expression in early embryos is a fundamental prerequisite for the obtainment of pregnancy. Eur J Immunol 32: 311-315, 2002.

25. Roussev RG, Keskintepe L and Coulam CB: Soluble HLA-G (sHLA-G) in human embryo culture media: a useful marker for assessing embryo quality and implantation potential for IVF. Fertil Steril 80: S12-S13, 2003.

26. Sher G, Keskintepe L, Nouriani M, Roussev R and Batzofin J: Expression of sHLA-G in supernatants of individually cultured 46-h embryos: a potentially valuable indicator of "embryo competency' and IVF outcome. Reprod Biomed Online 9: 74-78, 2004.

27. Criscuoli L, Rizzo R, Fuzzi B, Melchiorri L, Menicucci A, Cozzi C, Dabizzi S, Branconi F, Evangelisti P, Baricordi OR and Noci I: Lack of histocompatibility leukocyte antigen-G expression in early embryos is not related to germinal defects or impairment of interleukin-10 production by embryos. Gynecol Endocrinol 20: 264-269, 2005.

28. Noci I, Fuzzi B, Rizzo R, Melchiorri L, Criscuoli L, Dabizzi S, Biagiotti R, Pellegrini S, Menicucci A and Baricordi OR: Embryonic soluble HLA-G as a marker of developmental potential in embryos. Hum Reprod 20: 138-146, 2005.

29. Sher G, Keskintepe L, Batzofin J, Fisch J, Acacio B, Ahlering P, and Ginsburg M: Influence of early ICSI-derived embryo sHLA-G expression on pregnancy and implantation rates: a prospective study. Hum Reprod 20: 1359-1363, 2005. 
30. Sher G, Keskintepe L, Fisch JD, Acacio BA, Ahlering P, Batzofin J and Ginsburg M: Soluble human leukocyte antigen G expression in phase I culture media at $46 \mathrm{~h}$ after fertilization predicts pregnancy and implantation from day 3 embryo transfer. Fertil Steril 83: 1410-1414, 2005.

31. Yie SM, Balakier H, Motamedi G and Librach CL: Secretion of human leukocyte antigen-G by human embryos is associated with a higher in vitro fertilization pregnancy rate. Fertil Steril 83: 30-36, 2005

32. Desai N, Filipovits J and Goldfarb J: Secretion of soluble HLA-G by day 3 human embryos associated with higher pregnancy and implantation rates: assay of culture media using a new ELISA kit. Reprod Biomed Online 13: 272-277, 2006.

33. Fisch JD, Keskintepe L, Ginsburg M, Adamowicz M and Sher G: Graduated Embryo Score and soluble human leukocyte antigen-G expression improve assisted reproductive technology outcomes and suggest a basis for elective single-embryo transfer. Fertil Steril 87: 757-763, 2007.

34. Rebmann V, Switala M, Eue I, Schwahn E, Merzenich M and Grosse-Wilde H: Rapid evaluation of soluble HLA-G levels in supernatants of in vitro fertilized embryos. Human Immunol 68. 251-258, 2007

35. Shaikly VR, Morrison IE, Taranissi M, Noble CV, Withey AD, Cherry RJ, Blois SM and Fernández N: Analysis of HLA-G in maternal plasma, follicular fluid, and preimplantation embryos reveal an asymmetric pattern of expression. J Immunol 180: 4330-4337, 2008.
36. Van Lierop MJ, Wijnands F, Loke YW, Emmer PM, Lukassen HG, Braat DD, van der Meer A, Mosselman S and Joosten I: Detection of HLA-G by a specific sandwich ELISA using monoclonal antibodies G233 and 56B. Mol Hum Reprod 8: 776-784, 2002.

37. Sageshima N, Shobu T, Awai K, Hashimoto H, Yamashita M, Takeda N, Odawara Y, Nakanishi M, Hatake K and Ishitani A: Soluble HLA-G is absent from human embryo cultures: a reassessment of sHLA-G detection methods. J Reprod Immunol 75: 11-22, 2007.

38. Warner CM, Lampton PW, Newmark JA and Cohen J: Soluble human leukocyte antigen-G and pregnancy success. Reprod Biomed Online 17: 470-485, 2008.

39. Menezo Y, Elder K and Viville S: Soluble HLA-G release by the human embryo: an interesting artefact? Reprod Biomed Online 13: 763-764, 2006.

40. Apps R, Gardner L and Moffett A: A critical look at HLA-G. Trends Immunol 29: 313-321, 2008

41. Coticchio G, Sereni E, Serrao L, Mazzone S, Iadarola I and Borini A: What criteria for the definition of oocyte quality? Ann NY Acad Sci 1034: 132-144, 2004

42. Patrizio P, Fragouli E, Bianchi V, Borini A and Wells D: Molecular methods for selection of the ideal oocyte. Reprod Biomed Online 15: 346-353, 2007.

43. Liu Z, Shimada M and Richards JS: The involvement of the Toll-like receptor family in ovulation. J Assist Reprod Genet 25: 223-228, 2008. 